\title{
AiMT
}

Advances in Military Technology

Vol. 15 , No. 2, 2020, pp. 365-377

ISSN 1802-2308, eISSN 2533-4123

DOI 10.3849/aimt.01424

\section{The Method of Proactive Risk Assessment for Flight Safety Based on the Rate of Dangerous Events}

\author{
Y. Honcharenko ${ }^{1 *}$, O. Martyniuk ${ }^{1}$, O. Radko ${ }^{2}$ and P. Open'ko ${ }^{1}$ \\ ${ }^{1}$ National Defence University of Ukraine named after Ivan Cherniakhovskyi, Kyiv, Ukraine \\ ${ }^{2}$ National Aviation University, Kyiv, Ukraine
}

\begin{abstract}
The manuscript was received on 28 May 2020 and was accepted after revision for publication as research paper on 31 October 2020.
\end{abstract}

\begin{abstract}
:
This publication is devoted to the issues of risk assessment in the safety management system of military aviation. The method of proactive assessment of risks to flight safety based on the number of dangerous events, which is based on correlation-regression analysis, provides an opportunity to predict the conditions that contribute to the occurrence of accidents. Studies conducted with this method have confirmed the dependence of the number of aviation accidents and serious incidents on incidents recorded for different groups of reasons, as well as on the intensity of flight activities. The obtained results can provide initial data for quantitative risk assessment in the safety management system of military aviation. The implementation of such an approach is appropriate in safety management systems in the transition phase from active to proactive approaches, until a sufficient information database on hazards is accumulated.
\end{abstract}

\section{Keywords:}

correlation and regression analysis, flight safety, safety management system

\section{Introduction}

Flight operation of aircraft is related to the risks caused by many factors. The main factor, along with economic and political, affecting the safe operation of military aircraft, is the effectiveness of the safety management system (SMS). The main task of the SMS is to control and manage the risk factors. The components of risk factor management for flight safety (FS) are the identification of hazards, as well as the development and implementation of effective and adequate measures to reduce their negative impact [1].

\footnotetext{
* Corresponding author: Aviation Department, National Defence University of Ukraine named after Ivan Cherniakhovskyi, Povitroflotskyi Avenue, 28, Kyiv, Ukraine. E-mail: yev.v.honcharenko@gmail.com
} 
Air Force of Ukraine (AFU) is armed with aircrafts inherited from the USSR. Along with the aircrafts, reactive strategy for the management of FS was taken over, which is based on scientific works in the mid-80s of last century [2]. The essence of the reactive approach is to identify safety risks, assess them and take the necessary measures after a dangerous event (an accident, a serious incident (SI), an incident) occurred. This approach was logical and had economic expediency in the circumstances when the operating fleet was in the range of thousands of aircrafts (the amount of the USSR's Air Force fleet in 1990 was up to 7000 units). At that period, the Soviet Air Force was losing about 100 aircrafts per year (less than $1 \%$ of the aircraft fleet). Conditions of operation of military aircraft of Ukraine have other scales and features, but there are still negative trends in accidents (up to 2 accidents per year) [3]. The application of the reactive method of flight safety management of AFU does not allow responding adequately and in advance to existing and potential safety risks.

On the other hand, advanced aviation organizations such as International Civil Aviation Organization (ICAO), International Air Transport Association (IATA), The European Organisation for the Safety of Air Navigation (EUROCONTROL), World Flight Safety Fund (FSF) are constantly developing and improving more proactive, risk-based methods aimed at further reducing the number of aviation accidents [1, 4-7]. The essence of a proactive approach is early prevention, which is carried out by identifying hazardous factors (risk factors) and taking measures to reduce the risk before any dangerous event occurs. The use of scientifically developed methods of managing risk factors is a central component of a proactive approach [6].

ICAO documents $[5,6]$ indicate that each aviation entity defines the methods, indicators and criteria for the effectiveness of FS maintenance independently, in accordance with the conditions and peculiarities of aviation exploitation. NATO military aviation safety regulations, including the Royal Canadian Air Force, state that a proactive approach should be the basis of the Flight Safety Program [8]. It should be noted, that the introduction of advanced approaches to civil aviation risk management in the Royal Air Force of Canada is yielding positive results. Thus, over the past 50 years, the accident rate of Canadian military aircraft per 10000 hours of flight has decreased from 0.510 to 0.081 [9], which is one of the best results in the world.

Reduction of the number of accidents in the military aviation of Ukraine is possible by introducing a proactive approach to the SMS. However, up to now, no method, indicators and criteria for determining the effectiveness of FS have been developed. Therefore, the purpose of this publication is to develop a scientifically based method that will provide data which will predict potential accidents. Consequently, the data will enable to prevent the accidents from happening in accordance with the conditions and features of flight operation of aircraft of the AFU.

\section{Flight Safety Risk Assessment of Military Aviation}

The concept of risk by the international standard [4] is interpreted as the impact of uncertainty on the goals. In addition, the definition of risk is a combination of the consequences of events and the associated probability of events. ICAO documents define the risk in the field of FS as the presumed probability and severity of flight consequences [6]. It is almost impossible to predict the occurrence of an event in flight; however, having a sufficient database of observations of the results of flights can determine their intensity. The consequences of the hazards can be an accident, 
a serious incident, or an incident. Definitions of these events in the article are used in accordance with the terminology of ICAO [10].

The method of assessing the safety risk proposed by ICAO [6] involves the analysis of identified hazards. This requires the use of a comprehensive database of all hazards. Due to the small (compared to civil aviation) raid in military aviation, the manifestation of such risks will be a rare event, and the filling of an adequate database is a long process. The database of safety hazards is not maintained, but every dangerous event is recorded.

The classifier of incidents in the AFU identifies 15 types of their causes, which are grouped into three different groups:

- the first group - incidents related to incorrect (erroneous) actions, violations of rules by personnel,

- the second group - incidents related to failures of aircraft,

- the third group - incidents related to the influence of external factors that are could not be predicted.

Based on the database of flight operations in the AFU, the problem of risk assessment in the SMS will be solved by analyzing dangerous events and their intensity using the methods of mathematical statistics. To determine the dependence of the number of accident and SI on the number of incidents for different reasons, the method of correlation-regression analysis has been used. Therefore, it is necessary to determine the indicators and evaluation criteria.

The authors suggest that an increase in the number of incidents of a certain group of causes is an indication (signal) of the occurrence of conditions favorable for the commission of accident or SI. That is, the errors and violations committed by personnel have a greater impact on the number of accidents and the failures of aircraft on the number of SI. In addition, the number of accidents and SIs is affected by the intensity of flight activity.

The purpose of the authors' method of proactive safety risk assessment in military aviation is to determine the dependence of one FS indicator on another. The method has four steps, which can be conventionally designated as: I - Intensity, A - Average value, $\mathrm{C}$ - Correlation coefficient, $\mathrm{R}$ - Regression equation (IACR).

The initial data are absolute indicators of flight operation (flight hours, number of dangerous events, etc.).

In the first step, depending on the purpose of the assessment, based on the initial data of flight operation, it is determined by which indicators the assessment will be carried out. The following indicators are taken into account: type of dangerous events (accidents, SIs, incidents by types or groups of causes); time periods (year, month, etc.); types of aircraft (type of aviation: transport or tactical); level of assessment (Air Forces, aviation brigade, aviation squadron). After that, the intensity indicators $\lambda$ are calculated and it is determined between which indicators the dependence, i.e. forecasting rules, will be established.

The second step is to determine the average values of $\bar{\lambda}$, which will be used to calculate the sample correlation coefficients. In addition, based on the average values of the indicators $\bar{\lambda}$, the limits of the confidence intervals of these indicators $\left(\lambda_{\min }, \lambda_{\max }\right)$ are calculated as a criterion for the acceptable level of FS for these indicators. In addition, the sample standard deviation of the indicators is determined.

The third step is to calculate the correlation coefficients between the values of the evaluated indicators. 
In the fourth step, the values of the obtained correlation coefficients for adequacy (whether the indicators really correlate with each other) are checked, and then the regression equation of the dependence of one indicator on another is calculated, as well as the coefficient of determination.

Finally, diagrams of the scattering of values of indicators are constructed and conclusions are formulated.

The obtained results are the initial data for quantitative risk assessment in the SMS of military aviation. International Standard [11] provides guidance on the selection and application of a sufficient number of risk assessment methods in a wide range of situations. Estimation (ranking) of the consequences of the influence of dangerous factors within the method is carried out in accordance with the approach proposed in [7]: from A - catastrophic to E - insignificant. Risk levels (acceptable, satisfactory, and unacceptable) are represented in a $5 \times 5$ consequence/probability matrix. Detailed descriptions of the proposed technique are followed.

\subsection{Indicators for Assessing the Flight Safety of Military Aviation}

During theoretical research, the level of flight safety is quantified by the following indicators [2]: $Q$ - the level of risk (probability of accident); $P_{\mathrm{FS}}$ - the probability of accident absence (probability of FS); $t_{\text {acc }}-$ the average flight hours to the accident (SI, incident) for the analyzed period.

$$
Q=\frac{n_{\mathrm{acc}}}{N}, P_{\mathrm{FS}}=\mathrm{e}^{-n_{\mathrm{acc}}}, t_{\mathrm{acc}}=\frac{t_{\Sigma}}{n_{\mathrm{acc}}}
$$

where $n_{\text {acc }}$ - the number of accidents for a certain period of time, $N$ - the number of flights for a certain period, general flight hours $-t_{\Sigma}$.

In practice, in AFU, the analysis of flight safety is carried out by absolute data: the number of accidents, SIs and incidents, fixed during a certain period of operation (week, month, year, etc.); and relative - flight hours to dangerous event. Besides: general flight hours $-t_{\Sigma}$; the number of flights $-N$; the number of flight shift $-N_{\mathrm{fl}}$; the size of the aviation park (number of aircraft and helicopters) $-K_{\text {acft }}$.

Additionally, the authors propose to take into account intensity of flight activity. It is the relation of the general flight hours to the number of flight shift:

$$
t_{\text {int }}=\frac{t_{\Sigma}}{N_{\mathrm{fl}}}
$$

ICAO notes that events are usually not tracked in absolute values, but in the form of occurrence frequency [5]. The authors consider this approach to be the most rational, so the paper proposes not to analyze the time intervals between events, but the number of events per unit of time (intensity). The experience of AFU shows that the intensity of dangerous events differs by 100 or 1000 times, so for the convenience of analysis and perception of the results, it is proposed that each indicator determines its dimension, so that the values have no more than two digits after the comma, i.e.:

$$
\lambda_{\mathrm{acc}}=\frac{n_{\mathrm{acc}}}{t_{\Sigma}} \cdot 10^{5}, \lambda_{\mathrm{si}}=\frac{n_{\mathrm{si}}}{t_{\Sigma}} \cdot 10^{3}, \lambda_{\mathrm{inc}}=\frac{n_{\mathrm{inc}}}{t_{\Sigma}} \cdot 10^{2}, \lambda_{\text {inc1 }}=\frac{n_{\text {inc1 } 1}}{t_{\Sigma}} \cdot 10^{2}, \lambda_{\text {inc } 2}=\frac{n_{\text {inc } 2}}{t_{\Sigma}} \cdot 10^{2}(3)
$$

where $\lambda_{\text {acc }}$ - the accidents intensity - the number of accidents by $100000 \mathrm{~h}$ of flight hours; $\lambda_{\text {si }}-$ the SI intensity - the number of SI by $1000 \mathrm{~h}$ of flight hours; $\lambda_{\text {inc }}-$ the incidents intensity - the number of incidents by $100 \mathrm{~h}$ of flight hours; $\lambda_{\text {inc } 1}-$ the inten- 
sity of incidents of the first group - the number of incidents of the first reasons group by $100 \mathrm{~h}$ of flight hours; $\lambda_{\text {inc } 2}$ - the intensity of incidents of the second group - the number of incidents of the second reasons group by $100 \mathrm{~h}$ of flight hours; $n_{\mathrm{si}}-$ the number of SI; $n_{\text {inc }}$ - the number of incidents; $n_{\text {inc } 1}-$ the number of accidents of the first group; $n_{\text {inc } 2}$ - the number of accidents of the second group.

To conduct certain statistical researches using information releases on air events and incidents for 1996-2017 years (in AFU), by Eq. (3) the value of intensity of occurrences for years of exploitation has been calculated (Tab. 1).

Tab. 1 Dangerous events intensity for 1996-2017 years

\begin{tabular}{|c|c|c|c|c|c|c|c|}
\hline \multirow{2}{*}{ Year } & \multicolumn{3}{|c|}{ Dangerous events intensity } & \multirow{2}{*}{ Year } & \multicolumn{3}{c|}{ Dangerous events intensity } \\
& $\lambda_{\text {inc }}$ & $\lambda_{\text {si }}$ & $\lambda_{\text {acc }}$ & & $\lambda_{\text {inc }}$ & $\lambda_{\text {si }}$ & $\lambda_{\text {acc }}$ \\
\hline 1996 & 0.99 & 0.50 & 5.38 & 2007 & 0.94 & 1.32 & 0.00 \\
\hline 1997 & 1.24 & 0.61 & 2.44 & 2008 & 1.23 & 0.69 & 0.00 \\
\hline 1998 & 0.99 & 0.64 & 9.66 & 2009 & 1.04 & 1.01 & 0.00 \\
\hline 1999 & 1.18 & 0.72 & 6.57 & 2010 & 1.36 & 0.60 & 0.00 \\
\hline 2000 & 1.15 & 0.93 & 0.00 & 2011 & 1.29 & 1.79 & 0.00 \\
\hline 2001 & 2.08 & 1.49 & 0.00 & 2012 & 0.85 & 0.59 & 7.40 \\
\hline 2002 & 1.80 & 1.07 & 21.37 & 2013 & 1.13 & 0.54 & 0.00 \\
\hline 2003 & 1.26 & 0.82 & 0.00 & 2014 & 0.87 & 1.01 & 5.04 \\
\hline 2004 & 1.58 & 2.45 & 8.45 & 2015 & 1.26 & 0.96 & 5.99 \\
\hline 2005 & 1.35 & 0.93 & 0.00 & 2016 & 1.55 & 1.76 & 7.98 \\
\hline 2006 & 1.31 & 1.49 & 0.00 & 2017 & 1.49 & 1.23 & 7.70 \\
\hline
\end{tabular}

From Tab. 1 it can be concluded that the intensity of incidents and intensity of SIs have small distribution limits $\left(0.87 \leq \lambda_{\text {inc }} \leq 2.08 ; 0.50 \leq \lambda_{\text {si }} \leq 2.45\right)$. At the same time, the intensity of accidents varies in a wider range $\left(0 \leq \lambda_{\text {acc }} \leq 7.98\right)$ and is more random.

\subsection{Determination of Confidence Intervals of Flight Safety Indicators of Military Aviation}

To implement early prevention of accidents in the SMS, it is necessary to set thresholds, as well as the desired target levels for each indicator. They will serve as benchmarks for the unacceptable level of the indicator (intensity of dangerous events), or, conversely, the desired target (improved) level of frequency for such an indicator. As long as the trend does not go beyond certain limits, the number of such events will be considered acceptable (without deviations from the norm) for the relevant monitoring period.

To determine the limits of the acceptable level, a more appropriate method of the confidence interval was designed, which is calculated from observational data, and covers an unknown statistical parameter with a given reliability (confidence probability) [12]. We will assume that the probability of the indicator falling within this interval $\gamma=95 \%$ is sufficient for these studies. 
To determine the limits of the acceptable level of the indicator, its average value and confidence interval are calculated according to the method below.

The average value of the intensity of incidents is determined by the formula [12]:

$$
\overline{\lambda_{\text {inc }}}=\frac{1}{N_{\mathrm{c}}} \sum_{i=1}^{N_{\mathrm{c}}} \sum_{j=1}^{J} \lambda_{\text {inc } i}^{j}
$$

where $N_{\mathrm{c}}$ - the number of observations, $J$ - the number of causes of events (incidents), according to the classifier, $J=[1,15]$.

The sample standard deviation of accidents intensity:

$$
s d_{\mathrm{inc}}=\sqrt{\frac{1}{N_{\mathrm{c}}-1} \sum_{i=1}^{N_{\mathrm{c}}}\left(\lambda_{\mathrm{inc} i}-\overline{\lambda_{\mathrm{inc}}}\right)^{2}}
$$

Confidence interval of expected value $M\left[\lambda_{\text {inc }}\right]$ and variance of incidents intensity $\sigma\left[\lambda_{\text {inc }}\right]$ depending on the reliability (probability) $\gamma$ and degrees of freedom $n$ are determined by the formulas:

$$
\begin{gathered}
\overline{\lambda_{\text {inc }}}-t_{\gamma} \frac{s d}{\sqrt{N_{\mathrm{c}}}} \leq M\left[\lambda_{\text {inc }}\right] \leq \overline{\lambda_{\text {inc }}}+t_{\gamma} \frac{s d}{\sqrt{N_{\mathrm{c}}}} \\
s d_{\text {inc }}\left(1-q_{\gamma}\right) \leq \sigma\left[\lambda_{\text {inc }}\right] \leq s d_{\text {inc }}\left(1+q_{\gamma}\right)
\end{gathered}
$$

where $t_{\gamma}$ - the Student's distribution quantile $t_{\gamma}=f(\gamma, k), q_{\gamma}$ - the normal distribution quantile $q_{\gamma}=f(\gamma, k), k$ - the number of degrees of freedom.

The results of the calculations performed for Eqs (4)-(7) are shown in Tab. 2.

Tab. 2 Characteristics of confidence intervals of incident intensity depending on their causes

\begin{tabular}{|l|c|c|c|c|c|c|}
\hline \multirow{2}{*}{$\begin{array}{c}\text { Groups of causes of incident } \\
\text { intensity }\end{array}$} & \multicolumn{5}{|c|}{ Confidence interval parameters } \\
\cline { 2 - 8 } & $\lambda_{\text {inc }}^{-}$ & $s d$ & $\lambda_{\min }$ & $\lambda_{\max }$ & $\delta_{\min }$ & $\delta_{\max }$ \\
\hline the first group $-\lambda_{\text {inc1, }}\left[\mathrm{h}^{-1}\right]$ & 0.41 & 0.18 & 0.35 & 0.48 & 0.12 & 0.25 \\
\hline the second group $-\lambda_{\text {inc2, }}\left[\mathrm{h}^{-1}\right]$ & 0.77 & 0.25 & 0.68 & 0.86 & 0.16 & 0.33 \\
\hline the third group $-\lambda_{\text {inc3, }}\left[\mathrm{h}^{-1}\right]$ & 0.084 & 0.049 & 0.066 & 0.102 & 0.032 & 0.067 \\
\hline Total $-\lambda_{\text {inc }},\left[\mathrm{h}^{-1}\right]$ & $\mathbf{1 . 2 7}$ & $\mathbf{0 . 3 0}$ & $\mathbf{1 . 1 6}$ & $\mathbf{1 . 3 8}$ & $\mathbf{0 . 1 9}$ & $\mathbf{0 . 4 0}$ \\
\hline
\end{tabular}

From Tab. 2 it can be concluded that the highest intensity of incidents is recorded due to the failures of aircraft, as well as due to errors of aviation personnel. The limits of confidence intervals $\lambda$ indicated in the table should be used when assessing the level of flight safety for a certain period as a criterion for assessing the level of safety - the limits of acceptable levels. 


\subsection{Determination of the Correlation Between Flight Safety Indicators of Military Aviation}

Aviation events and incidents (failures of aircraft, aviation personnel errors, and the influence of external factors) are random events. As a rule, there can be a stochastic relationship between random variables, during which the distribution of another quantity $Y$ changes with the change of the quantity $X$. The authors assume that the dependence between $X$ and $Y$ is linear. The most well-known measure of the linear dependence of the two quantities is the Pearson correlation coefficient.

Estimation of the dependence of the number of accidents and SIs on the number of incidents for different groups of reasons will be performed by the method of correlation analysis. To apply this method, the following conditions must be met: the sample must be representative, i.e. the number of observations must be sufficient; the parameters under study should be mutually independent and distributed according to the normal law [12].

Within the framework of this work, we will consider the results of all flight activities from 1992 to the present - the general set of events that occurred in the aviation of the AFU. Indicators of this activity for 22 years (from 1996 to 2017) can be considered as a representative sample.

The distribution law of intensity of incidents $\lambda_{\text {inc }}$ is defined graphically - on the histogram of this quantity size. To construct a histogram of the intensity of incident intensities $\omega_{\text {inc }}$, it is necessary to determine the number of intervals of the histogram $K$ (according to the Sturges rule).

$$
K=1+\left\lfloor\log _{2} N_{\mathrm{c}}\right\rfloor
$$

where symbols \lfloor\rfloor represent floor function.

Since for $N_{\mathrm{c}}=22$ years, $\mathrm{K}=7$; the value of $\lambda_{\text {inc }}$ is in the range of $0.87 \leq \lambda_{\text {inc }} \leq 2.08 ;$ step interval $\Delta l_{\text {inc }}$ for density $\omega_{\text {inc }}$, calculated:

$$
\Delta l_{\text {inc }} \approx \frac{\lambda_{\text {inc } \_ \text {max }}-\lambda_{\text {inc_min }}}{K}
$$

The results of the calculations can be $\Delta l_{\text {inc }}=0.2$. Using the data from Tab. 1 and by Eqs (8)-(9), the following histogram of incidents intensity was constructed (Fig. 1).

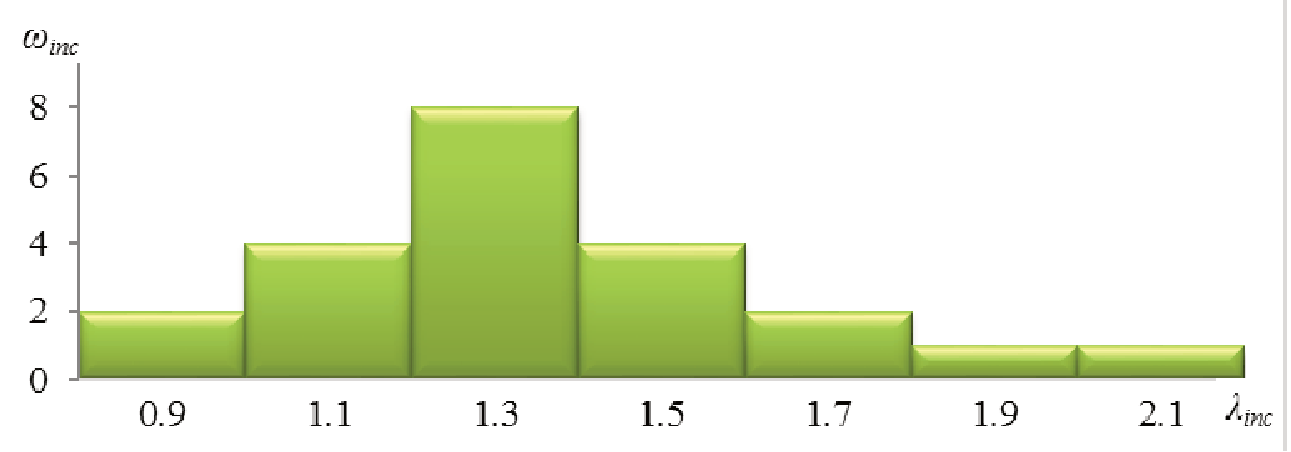

Fig. 1 Histogram of incidents intensity

According to the form of the histogram of the incidents intensity we can conclude that the general set of this random variable is distributed according to the law close to 
normal. Therefore, the fulfillment of conditions [12] to determine the relationship between indicators allows the use of correlation analysis.

A necessary and sufficient condition for the correlation of random variables $X$ and $Y$ is the inequality of zero correlation coefficient of the general set $X$ and $Y$ [12]:

$$
\rho_{x y}=\frac{M\{(X-M[X])(Y-M[Y])\}}{\sqrt{D[X] D[Y]}}
$$

where $M[X], M[Y]$ - the expected value $X$ and $Y, D[X], D[Y]$ - dispersion $X$ and $Y$.

Because according to the results of observations it is impossible to find $\rho_{x y}$, in practice, let us consider a sample correlation coefficient $r_{x y}$ :

$$
r_{x y}=\frac{\sum\left(X_{i}-\bar{X}\right)\left(Y_{i}-\bar{Y}\right)}{\sqrt{\sum\left(X_{i}-\bar{X}\right)^{2} \sum\left(Y_{i}-\bar{Y}\right)^{2}}}
$$

According to Eq. (11), pairwise Pearson correlation coefficients of flight safety indicators have been calculated. The results of the calculations are summarized in the matrix below (Tab. 3).

Tab. 3 Matrix of Pearson correlation coefficients of flight safety indicators of the Air Force of Ukraine

\begin{tabular}{|c|c|c|c|c|c|c|c|c|c|c|c|c|c|c|}
\hline$X$ & $t_{\Sigma}$ & $N_{\mathrm{fl}}$ & $K_{\text {acft }}$ & $t_{\text {int }}$ & $n_{\text {inc }}$ & $n_{\text {inc1 }}$ & $n_{\text {inc2 }}$ & $n_{\text {si }}$ & $n_{\text {acc }}$ & $\lambda_{\text {inc }}$ & $\lambda_{\text {inc1 }}$ & $\lambda_{\text {inc2 }}$ & $\lambda_{\text {si }}$ & $\lambda_{\text {acc }}$ \\
\hline$t_{\Sigma}$ & 1 & 0.84 & 0.79 & 0.78 & $\mathbf{0 . 9 6}$ & 0.94 & 0.84 & $\mathbf{0 . 7 1}$ & $\mathbf{0 . 7 4}$ & -0.32 & 0.35 & -0.57 & -0.42 & 0.19 \\
\hline$N_{\text {fl }}$ & 0.84 & 1 & 0.79 & 0.41 & $\mathbf{0 . 8 5}$ & 0.71 & $\mathbf{0 . 9}$ & $\mathbf{0 . 7 2}$ & $\mathbf{0 . 6 5}$ & -0.21 & 0.09 & -0.28 & -0.28 & 0.27 \\
\hline$K_{\text {acft }}$ & 0.79 & 0.79 & 1 & 0.66 & 0.86 & 0.76 & $\mathbf{0 . 8 7}$ & $\mathbf{0 . 7 9}$ & $\mathbf{0 . 6 8}$ & 0.08 & 0.46 & -0.2 & -0.15 & 0.37 \\
\hline$t_{\text {int }}$ & 0.78 & 0.41 & 0.66 & 1 & 0.72 & $\mathbf{0 . 8}$ & 0.52 & $\mathbf{0 . 5 2}$ & $\mathbf{0 . 5 6}$ & -0.31 & $\mathbf{0 . 5 4}$ & -0.66 & -0.4 & 0.1 \\
\hline$n_{\text {inc }}$ & 0.96 & 0.85 & 0.86 & 0.72 & 1 & 0.95 & 0.91 & 0.77 & 0.7 & -0.07 & 0.49 & -0.38 & -0.3 & 0.24 \\
\hline$n_{\text {inc1 }}$ & 0.94 & 0.71 & 0.76 & 0.8 & 0.95 & 1 & 0.75 & 0.66 & $\mathbf{0 . 6 8}$ & -0.14 & 0.62 & -0.55 & -0.37 & 0.17 \\
\hline$n_{\text {inc2 }}$ & 0.84 & 0.9 & 0.87 & 0.52 & 0.91 & 0.75 & 1 & $\mathbf{0 . 8 2}$ & 0.63 & 0.06 & 0.26 & -0.09 & -0.14 & 0.3 \\
\hline$n_{\text {si }}$ & 0.71 & 0.72 & 0.79 & 0.52 & 0.77 & 0.66 & 0.82 & 1 & 0.61 & 0.04 & 0.32 & -0.12 & 0.28 & 0.32 \\
\hline$n_{\text {acc }}$ & 0.74 & 0.65 & 0.68 & 0.56 & 0.7 & 0.68 & 0.63 & 0.61 & 1 & -0.14 & 0.3 & -0.34 & -0.22 & 0.72 \\
\hline$\lambda_{\text {inc }}$ & -0.32 & -0.21 & 0.08 & -0.31 & -0.07 & -0.14 & 0.06 & 0.04 & -0.14 & 1 & 0.44 & 0.81 & 0.5 & 0.23 \\
\hline$\lambda_{\text {inc1 }}$ & 0.35 & 0.09 & 0.46 & 0.54 & 0.49 & 0.62 & 0.26 & 0.32 & $\mathbf{0 . 3}$ & 0.44 & 1 & -0.15 & 0 & 0.19 \\
\hline$\lambda_{\text {inc2 }}$ & -0.57 & -0.28 & -0.2 & -0.66 & -0.38 & -0.55 & -0.09 & -0.12 & -0.34 & 0.81 & -0.15 & 1 & 0.6 & 0.15 \\
\hline$\lambda_{\text {si }}$ & -0.42 & -0.28 & -0.15 & -0.4 & -0.3 & -0.37 & -0.14 & 0.28 & -0.22 & 0.5 & 0 & 0.6 & 1 & 0.09 \\
\hline$\lambda_{\text {acc }}$ & 0.19 & 0.27 & 0.37 & 0.1 & 0.24 & 0.17 & 0.3 & 0.32 & 0.72 & 0.23 & 0.19 & 0.15 & 0.09 & 1 \\
\hline
\end{tabular}

From the matrix (Tab. 3), we can conclude that the following quantitative indicators confirm the authors' assumption about the dependence of the number of accidents and SIs on dangerous factors of a certain group of reasons.

Number of accidents is more influenced by:

- total flight hours $\left(r_{x y}=0.74\right)$, 
- number of incidents of the first group of causes $\left(r_{x y}=0.68\right)$ and the intensity of these incidents $\left(r_{x y}=0.3\right)$; this is due to the fact that the cause of $80 \%$ of accidents is the so-called "human factor",

- intensity of flight activity $\left(r_{x y}=0.56\right)$.

Number of SIs depends on:

- number of incidents of the second group of causes $\left(r_{x y}=0.82\right)$ which is due to the fact that most SIs are accounted for due to aircraft failures,

- fleet of aircraft $\left(r_{x y}=0.79\right)$,

- number of flight shift $\left(r_{x y}=0.72\right)$.

The number of reported incidents $\left(r_{x y}=0.96\right)$, including SIs $\left(r_{x y}=0.71\right)$, and the number of accidents $\left(r_{x y}=0.74\right)$ depend on the total flight hours.

The number of incidents of the second group of causes $\left(r_{x y}=0.9\right)$, the total number of incidents $\left(r_{x y}=0.85\right)$ as well as SIs $\left(r_{x y}=0.72\right)$ and the number of accidents $\left(r_{x y}=0.65\right)$ depend on the number of flight shifts.

The number of reported incidents $\left(r_{x y}=0.86\right)$, including the second group incidents $\left(r_{x y}=0.87\right)$, the number of accidents $\left(r_{x y}=0.68\right)$ depend on the number of the fleet of aircraft involved in the flights.

The number of incidents of the first group $\left(r_{x y}=0.8\right)$ depend on the intensity of flight activity. It is due to the fact that this increases the load on aviation personnel, which in these conditions makes more mistakes. To a lesser extent, $t_{\text {int }}$ affects the amount of accidents $\left(r_{x y}=0.56\right)$ and the amount of SIs $\left(r_{x y}=0.52\right)$.

To confirm the assumption of a linear relationship between the indicators of FS, the values of Spearman's rank correlation (nonlinear) coefficient were calculated for three pairs of indicators (Tab. 4).

Tab. 4 Comparative table of Pearson's linear correlation coefficients and Spearman's rank correlation

\begin{tabular}{|c|c|c|c|}
\hline$X$ & $Y$ & Pearson's $r_{x y}$ & Spearman's $r_{x y}$ \\
\hline$N_{f l}$ & $n_{\text {inc } 2}$ & 0.9 & 0.32 \\
\hline$t_{i n t}$ & $n_{\text {incl }}$ & 0.8 & 0.74 \\
\hline$t_{\text {int }}$ & $n_{\text {acc }}$ & 0.56 & 0.32 \\
\hline
\end{tabular}

Tab. 4 shows that the values of the Pearson correlation coefficients are closer to 1 than the Spearman rank correlation. This means that the correlation between the indicators is rather linear than curve.

In this section, the values of the stochastic relationship between the indicators of FS were obtained. Next, to determine the mathematical model of this relationship, regression analysis should be used.

\subsection{Regression Analysis of Flight Safety Indicators of Military Aviation}

Regression analysis makes it possible to determine the functions of the dependence of the number of accidents and SIs on the number of the first or second group incidents. This allows you to predict the occurrence of conditions that contribute to the emergence of an SI or accident. The linear regression equation has the form:

$$
Y=\beta X+\alpha
$$

where $\alpha$ is the intercept, $\beta$ is the regression coefficient. 
To construct the regression line (12) it is necessary to determine the mean value of the number of dependent events (accident or SI) $\bar{Y}$ and the prerequisites $\bar{X}$, "corrected", i.e. unbiased standard deviations $s d_{y}$ and $s d_{x}$ and the estimate of the correlation coefficient $r_{x y}$.

The essence of the method of constructing a regression line will be considered based on the proposed assumption about the dependence of the number of accidents on the intensity of flight activity.

The first step is to calculate the values required to determine the dependence of the number of accidents $\left(Y=n_{\text {acc }}\right)$ on the intensity of flight activity $\left(X=t_{\text {int }}\right)$. According to Eqs (5), (6), and (11), we obtain $\bar{X}=22.54 ; \bar{Y}=0.77 ; s d_{x}=8.26 ; s d_{y}=0.82$; $r_{x y}=0.56$.

The significance of the sample correlation coefficient $r_{x y}$ is checked by making a null hypothesis about the equality of the zero correlation coefficient of the general population $\rho_{x y}$. To check it, a special description is made [13]:

$$
T_{x y}=\frac{r_{x y} \sqrt{N_{\mathrm{c}}-2}}{\sqrt{1-r_{x y}^{2}}}
$$

and it is compared with the tabular critical value $t_{\gamma}$ (which is calculated according to the table of critical points of the Student's distribution [13] in accordance with the given level of significance $\alpha_{\gamma}=1-\gamma$ and the number of degrees of freedom $k=N_{\mathrm{c}}-2$ ).

If $\left|T_{x y}\right|<t_{\gamma}$ - there is no reason to reject the null hypothesis, therefore, there is no correlation between random variables $X$ and $Y$.

If $\left|T_{x y}\right|>t_{\gamma}$ - the null hypothesis is rejected, therefore, the sample correlation coefficient $r_{x y}$ is significantly different from zero, i.e. $X$ and $Y$ are correlated.

By Eq. (13) we obtain $T_{x y}=3.03$. The value of $t_{\gamma}$ is calculated for the confidence probability $\quad \gamma=95 \%, \quad \alpha_{\gamma}=1-0.95=5 \% ; \quad$ at $\quad N_{\mathrm{c}}=22, \quad k=N_{\mathrm{c}}-2=20$; $t_{\gamma}(0.05 ; 20)=2.086$. The condition is fulfilled $\left|T_{x y}\right|>t_{\gamma}$, i.e. the intensity of flight activity $-X$ and the number of accidents $-Y$ are correlated random variables. sion:

The second step is to determine the coefficient and the free member of the regres-

$$
\beta=r_{x y} \frac{s d_{y}}{s d_{x}}, \quad \alpha=\overline{Y-} \beta \bar{X}
$$

According to Eqs (12) and (14), we obtain: $\beta=0.0661, \alpha=-0.7175$. In addition, the equation of linear correlation has the form: $Y=0.0661 X-0.7175$.

Fig. 2 shows the scattering diagram (correlation graph) between the intensity of flight activity $\left(t_{\text {int }}\right)$ and the number of accidents $\left(n_{\text {acc }}\right)$ with the line of dependence. The coefficient of determination $R^{2}=r_{x y}{ }^{2}$ shows the degree of correspondence of the proposed model to the real dependence between variables. The closer the value of the coefficient to 1 , the stronger the dependence. For acceptable models, $R^{2}$ is assumed to be more than 0.5 (or $50 \%$ ). In our case, $R^{2}=0.3152$. That is, the number of accidents depends on the intensity of flight activity only by $31.52 \%$.

The use of this algorithm makes it possible to determine the mathematical dependence of one indicator on another one for any pair of Tab. 3. The authors calculated the dependences and plotted the correlations with regression equations and coefficients of determination for: the number of incidents of the first group from the intensity of flight activity; the number of accidents from the number of incidents of the 
first group; the number of SIs from the number of incidents of the second group (Figs $3,4,5)$.

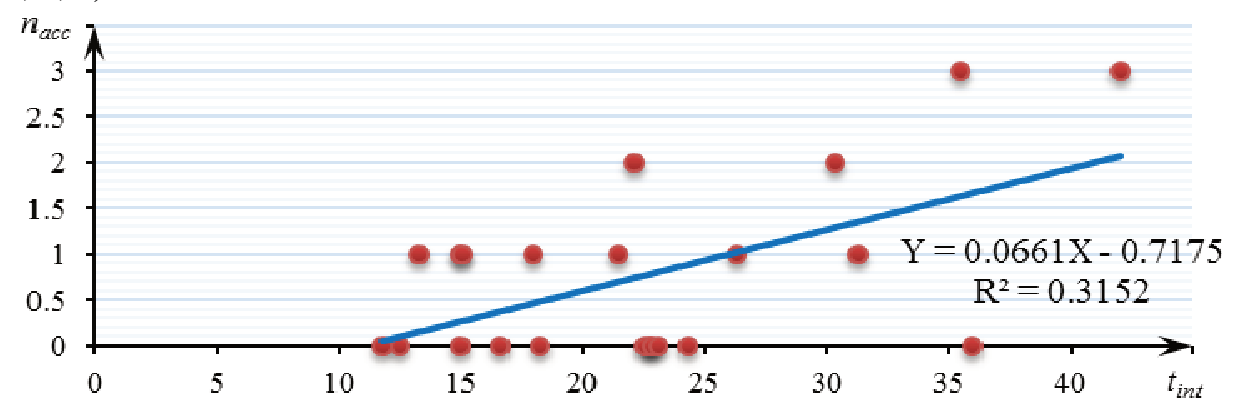

Fig. 2 Dependence of the number of accidents on the intensity of flight activity

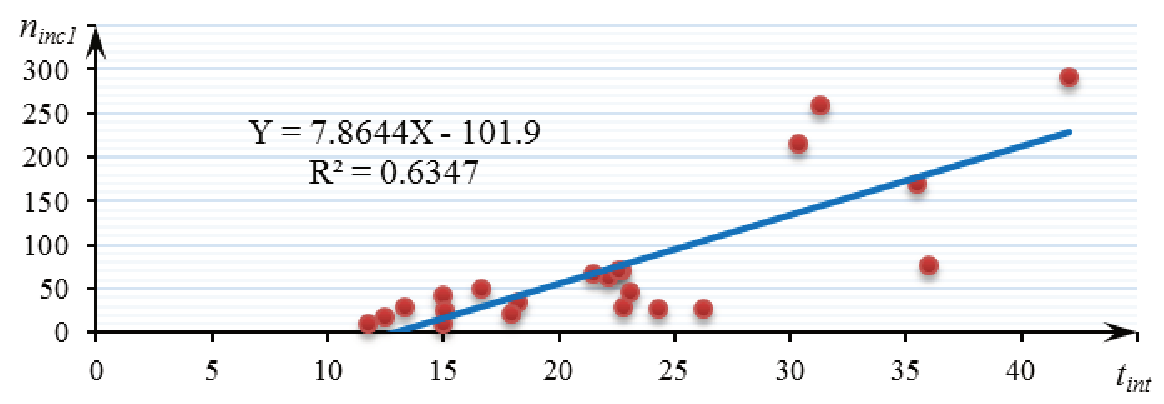

Fig. 3 Dependence of the number of incidents of the first group on the intensity of flight activity

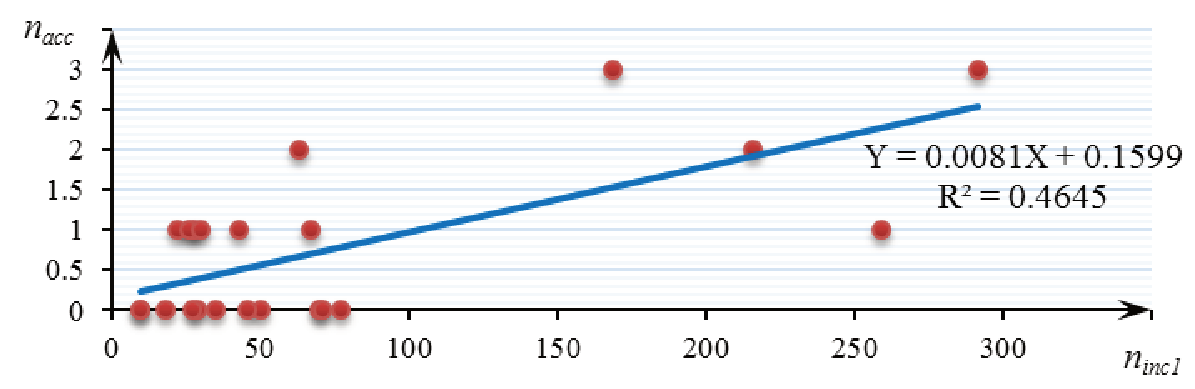

Fig. 4 Dependence of the number of accidents on the number of incidents of the first group

The equation of the dependence of the number of the first group incidents on the intensity of flight activity $-Y=7.8644 X-101.9$. The percentage of reliability of this forecast $R^{2}=0.6347$, i.e. $63.47 \%$.

The number of accidents in $46.45 \%$ is affected by the number of incidents of the first group, mathematical model of this connection $Y=0.0081 X+0.1599$.

The number of SIs in $67.07 \%$ is influenced by the number of incidents of the second group of reasons, the mathematical model of this connection $\mathrm{Y}=0.1153 \mathrm{X}+2.1327$ 


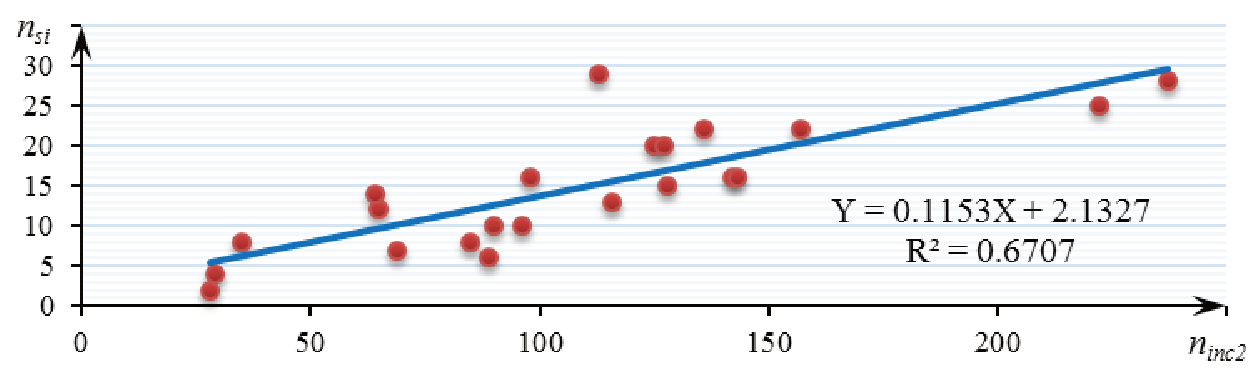

Fig. 5 Dependence of the number of SIs on the number of incidents of the second group

The use of the method of regression analysis allows predicting the occurrence of conditions that contribute to the occurrence of SIs or accidents, and the degree of reliability of this prediction is characterized by value $R^{2}$.

\section{Conclusions}

The method of proactive assessment of risks to flight safety for military aviation by the number of dangerous events, which is based on correlation-regression analysis, provides an opportunity to predict the occurrence of conditions that contribute to the emergence of an SI or accident. The obtained results, in turn, will be the initial data for quantitative risk assessment in the SMS of military aviation. The introduction of such an approach is appropriate in SMS in the transition from active to proactive approaches, until a sufficient information database on hazardous factors is accumulated. The presented method makes it possible to take timely measures to prevent accidents, which will increase the efficiency of the FS.

Studies conducted by using the IACR method have confirmed the assumption of the dependence of the number of accidents and SIs on incidents recorded for different groups of reasons, as well as on the intensity of flight activities. They show that the number of accidents in $46.45 \%$ is affected by the number of incidents of the first group of causes (human factors) and in $31.52 \%$ the intensity of flight activity. The number of SIs in $67.07 \%$ is influenced by the number of incidents of the second group of causes (failures of aircraft). The number of incidents of group 1 depends on the intensity of flight activities by $63.47 \%$.

As a criterion for the assessment of the FS level - the bounds of acceptable levels - it is proposed to use the limits of confidence intervals of incident intensity $\left(\lambda_{\min }\right.$, $\left.\lambda_{\max }\right)$.

The direction of further research may be determining the best method of risk assessment, as well as the use of the application of Fuzzy Logic methods in determining the effectiveness of FS (the construction of membership functions of FS and the definition of a set of fuzzy rules). In addition, to improve the reliability of the results obtained when calculating the values of FS indicators according to this method, it is advisable to consider the types of aircraft, because the conditions of their flight operation (average flight hours, total flight time, etc.) are different. 


\section{References}

[1] Annex 19 to the Convention on International Civil Aviation - Safety Management. $2^{\text {nd }}$ ed. Montreal: International Civil Aviation Organization, 2016.

[2] ZHULEV, V.I. and IVANOV, V.S. Flight Safety of Aircraft (in Russian). Moscow: Transport, 1986. 224 p. ISBN 978-5-45-834929-1.

[3] HONCHARENKO, Y.V. Flight Safety Culture of the State Aviation of Ukraine (in Ukrainian). Science and Defence, 2019, no. 1, p. 36-39. DOI 10.33099/26181614-2019-6-1-36-39.

[4] ISO 31000:2018, Risk management - Guidelines.

[5] Doc 9859-AN/474, Safety Management Manual, $3^{\text {rd }}$ ed. Montreal: ICAO, 2013.

[6] Doc 9859-AN/474, Safety Management Manual, $4^{\text {th }}$ ed. Montreal: ICAO, 2017.

[7] MARTYNIUK, O., RADKO, O. and HONCHARENKO, Y. The Risk Assessment in the State Aviation Safety Performance System (in Ukrainian). Modern Information Technologies in the Sphere of Security and Defence, 2019, vol. 34, no. 1, p. 155-160. DOI 10.33099/2311-7249/2019-34-1-155-160T.

[8] A-GA-135-001/AA-001 Flight Safety for the Canadian Armed Forces [online]. December 2018. [viewed 2020-05-20]. Available from: http://www.rcafarc.forces.gc.ca/en/flight-safety/manuals.page

[9] The Royal Canadian Air Force Highlights Historical Lows of Flight Safety Incidents During the Air Forces Flight Safety Committee (Europe) Meeting [online]. July 2019. [viewed 2020-05-20]. Available from: https://www.canada.ca/en/ department-national-defence/news/2019/07/the-royal-canadian-air-forcehighlights-historical-lows-of-flight-safety-incidents-during-the-air-forces-flightsafety-committee-europe-meeting.html

[10] Aircraft Accident and Incident Investigation - Annex 13 to the Convention on International Civil Aviation, $10^{\text {th }}$ ed. Montreal: ICAO, 2010.

[11] IEC 31010:2019, Risk Management - Risk Assessment Techniques.

[12] MARMOZA, A.T. Theory of Statistics (in Ukrainian). Kyiv: Tsentr Uchbovoi Literatury, 2013. 592 p. ISBN 978-617-673-163-4.

[13] KREMER, N.Sh. Probability Theory and Mathematical Statistics (in Russian). $2^{\text {nd }}$ ed. Moscow: UNITI, 2007. 573 p. ISBN 978-5-238-01101-1. 